\title{
Noradrenergic Modulation of the Masseteric Reflex in Behaving Cats. II. Physiological Studies
}

\author{
Ines L. Stafford and Barry L. Jacobs \\ Program in Neuroscience, Department of Psychology, Princeton University, Princeton, New Jersey 08544
}

Studies in the preceding paper demonstrated that the amplitude of the masseteric reflex in behaving cats is augmented by pharmacological manipulations that increase norepinephrine (NE) tone in the motor trigeminal nucleus (MoV) through exogenous means. The present studies examine whether such a relationship also exists under physiological conditions, i.e., whether physiological increases in NE synaptic activity are correlated with increases in the reflex amplitude. The masseteric reflex was elicited in behaving cats by electrical stimulation of the mesencephalic trigeminal nucleus (MesV) and the response recorded via electrodes permanently placed in the masseter muscle. Following baseline measures of the reflex amplitude, the reflex was again elicited while cats were exposed to various environmental stimuli known to activate NE neurons: $15 \mathrm{~min}$ of $100-\mathrm{dB}$ white noise, confrontation with a dog, or auditory clicks presented repetitively at various intervals prior to MesV stimulation. Presentation of the white noise or the dog significantly facilitated the reflex response for the duration of the exposure. The clicks produced reflex facilitation at 100 and $150 \mathrm{msec}$ following their presentation and reflex suppression at 20 msec. Two approaches were then employed to determine whether NE mediated, at least in part, augmentation of the reflex produced by these environmental conditions. In the first, cats were given either the $\alpha$-1-noradrenergic antagonist prazosin (5 mg/kg, i.p.) or the serotonin antagonist methysergide $(0.5 \mathrm{mg} / \mathrm{kg}$, i.p.). In all cases, prazosin blocked the reflex augmentation whereas methysergide was without effect. In the second study, directed at anatomically localizing these effects, the catecholamine neurotoxin 6-hydroxydopamine $(4 \mu \mathrm{g}$ in $1 \mu \mathrm{l})$ was infused unilaterally into the MoV to produce denervation of the NE input to the motor component of the reflex arc. One week later, the reflex was tested on both the intact and denervated sides while the cat was re-exposed to the aforementioned conditions. The normal augmentation was either blocked or markedly diminished on the denervated side while remaining unchanged on the intact side. In both the pharmacological and neurotoxin experiments the suppression produced by the 20 -msec click-reflex

Received Mar. 7, 1989; revised June 6, 1989; accepted June 23, 1989.

This work was funded by the U.S. Air Force (AFOSR 87-0301-A) and the NIMH (MH-23433). We thank Lynn Wilkinson for her invaluable assistance with the neurochemical assays, Drs. David Morilak and Casimir Fornal for numerous helpful discussions and advice, and Christine Metzler for excellent technical support.

Correspondence should be addressed to Ines L. Stafford-Segert, Brain Research Institute, Center for Health Sciences 43-385, UCLA, Los Angeles, CA 90024.

Copyright (C) 1990 Society for Neuroscience 0270-6474/90/010099-09\$02.00/0 interval was maintained, indicating that these effects were not attributable to nonspecific damage. These data represent the first demonstration that the release of NE, at a specific site and under physiological conditions, facilitates behavioral output in the intact organism. Furthermore, the neurotoxin experiment goes beyond correlation and establishes that a cause-and-effect relationship exists between increased NE activity and augmentation of the reflex response.

The experiments described in the preceding paper demonstrated that the amplitude of the masseteric reflex was augmented by pharmacological manipulations that increase norepinephrine (NE) tone in the motor trigeminal nucleus (MoV) through exogenous means. A more important question is whether such a relationship holds under physiological conditions, i.e., whether increases in NE synaptic activity are correlated with increases in the reflex amplitude.

In the present experiment, we examined whether the masseteric reflex is facilitated by conditions that are known to physiologically alter NE neuronal activity. Previous data that are consistent with this hypothesis show that the amplitude of the reflex in unanesthetized cats changes across the stages of the sleep-wake cycle (Chase et al., 1968) in a manner paralleling the activity of NE neurons (Rasmussen et al., 1986; Reiner, 1986). The amplitude of the masseteric reflex is largest during periods of active wakefulness, decreases during slow wave sleep, and is smallest during periods of REM sleep. NE neuronal activity exhibits a similar state-related pattern, showing the highest discharge rate during the active waking state, progressively decreasing as the cat enters quiet waking and slow-wave sleep states, and, finally, virtually ceasing to discharge during REM sleep. In addition to changes across the sleep-wake cycle, the activity of NE neurons is affected by a number of other factors. Single-unit studies indicate that a number of environmental stimuli and physiological variables cause an increase in the discharge rate of NE neurons in the locus coeruleus (LC) of the freely moving cat (for reviews, see Foote et al., 1983; Jacobs, 1986). Among the conditions shown to be effective in activating LC-NE neurons in cats are sensory stimuli such as clicks or light flashes, which produce phasic activation (Rasmussen et al., 1986), and stressors such as exposure to $100-\mathrm{dB}$ white noise or confrontation with a dog, which produce tonic activation (Abercrombie and Jacobs, 1987; Levine et al., 1987).

The NE innervation of MoV arises from the lateral tegmental NE cell groups (Vornov and Sutin, 1983; Grzanna et al., 1987). To date, the conditions that activate lateral tegmental NE neurons have not been well characterized. However, lateral teg- 
mental NE neurons do show state-related changes in discharge rate similar to those of LC neurons (Reiner, 1986). In addition, pilot studies from our laboratory suggest that lateral tegmental neurons are activated by the same stimuli as LC-NE neurons.

Increased discharge of NE neurons in response to the above conditions suggests a corresponding increase in NE release at axon terminal sites. This is supported directly by neurochemical studies showing that NE turnover in the brain is increased by exposing animals to various stressors (Tanaka et al., 1983; Glavin, 1985). If increased NE release in the brain is responsible for increases seen in the masseteric reflex, then one would predict that those conditions which activate NE neurons should also augment the masseteric reflex.

This hypothesis was tested in the present series of studies. It was predicted that the amplitude of the masseteric reflex in behaving cats would be increased during exposure to stimuli known to activate NE neurons (e.g., exposure to loud white noise, confrontation with a dog, and repetitive presentations of a click). Moreover, it was predicted that the facilitation would be blocked or at least attenuated by administration of adrenergic antagonist drugs and by selective destruction of NE terminals in MoV. The latter experiments, if successful, would transform the relationship between NE neuronal activity and augmentation of the reflex from one of simple correlation to one of cause and effect.

\section{Materials and Methods}

\section{Subjects and basic recording procedure}

Adult cats were prepared for masseteric recording as in the preceding paper. Briefly, the reflex was elicited by electrical stimulation of the sensory afferent cell bodies in the mesencephalic trigeminal nucleus (MesV) by means of permanently implanted bipolar electrodes. Reflex amplitude was measured by means of Teflon-coated stainless steel bipolar recording electrodes placed in the ipsilateral masseter muscle. On the day of an experiment, the subject was placed in the experimental booth and baseline current-response curves were determined as previously described. The reflex was elicited at each of 3 different current levels by delivering square-wave pulses to MesV once every $6 \mathrm{sec}$, for a total of 8 trials at each level. The highest and lowest values at each current level were discarded; a mean reflex response amplitude was then calculated from the remaining 6 values. This process was repeated, and when the 2 values determined for each current were stable $( \pm 10 \%)$, the 2 means were averaged to create a baseline value. The experimental manipulations (described in detail below) were then introduced and the reflex elicited at various time points during and after the manipulation. For the first series of studies, the reflex was elicited unilaterally and the response amplitude measured ipsilaterally. For the second, the reflex was elicited and measured bilaterally.

As in the preceding paper, behavioral state was carefully monitored during the experimental sessions. All tests were conducted when the subject was judged to be in a quiet waking state, as evidenced by a stable tonic EMC, infrequent or no eye movements, and no gross bodily movements. This issue is also addressed in more detail in the Discussion section of this paper.

\section{Environmental manipulations}

White noise. A Grason-Stadler Model 901B Noise Generator was adjusted to emit white noise of $100 \mathrm{~dB}$ intensity via a $10 \times 20 \mathrm{~cm}$ speaker installed in a wall of the recording chamber $(65 \times 65 \times 95 \mathrm{~cm}$ with a clear Plexiglas door). Following determination of baseline amplitude values at the 3 current levels, subjects were exposed to this stimulus for $15 \mathrm{~min}$. The reflex measurements were then begun at $\min 1,5$, and 10 of the white noise presentation and again 1 and $15 \mathrm{~min}$ after its termination.

Dog. An adult dog was introduced into the room housing the recording chamber. A wire mesh screen separated the cat from the dog, thus allowing visual, auditory, and olfactory cues to be sensed. The dog was leashed and held in front of the mesh screen, facing the cat, for $5 \mathrm{~min}$.
The reflex was tested at 3 current levels before, at the onset of the $5 \mathrm{~min}$ dog exposure, and again 1 and $15 \mathrm{~min}$ after removal of the dog.

Click. Two Grass stimulators were programmed to first deliver a single click ( $113 \mathrm{~dB}, 1$-msec-duration square-wave pulses) through the speaker in the wall of the chamber and then, following selected intervals, to deliver MesV stimulation. The intervals between click presentation and MesV stimulation were 0, 20,50,100,150, 200, 250, 500, and 1000 msec. These intervals were chosen for several reasons. Previous work in our laboratory (Rasmussen et al., 1986) indicated that NE neurons in cat are activated by clicks at a latency of $\sim 50 \mathrm{msec}$, and for a duration of $\sim 100 \mathrm{msec}$. Therefore, it was predicted that reflex facilitation should be produced only by clicks preceding stimulation within a particular temporal window (i.e., $\sim 50-150 \mathrm{msec}$ ). Clicks preceding MesV stimulation by longer intervals, or simultaneously, should be ineffective. The $20-\mathrm{msec}$ interval was included because pilot runs indicated that the reflex amplitude was suppressed at this time point, thereby providing a control. Each click-stimulation pair was presented in random order, for a total of 12 trials at each interval. In addition, the reflex was measured before and after click presentation. All trials were conducted only at the low current level in order to minimize possible habituation effects from repetitive stimulus presentations. The amplitude values at each interval were averaged and compared with the pre- and postclick means.

\section{Drug studies}

Antagonists and neurotoxin lesions. Two approaches were used in order to determine whether the changes in the masseteric reflex produced by these environmental conditions were causally related to activation of NE neurons. Because, as described in the preceding paper, the results were not differentially affected as a function of current level, all tests in both series were conducted using only the low current level. In the first series, the response to white noise or click was tested in cats that were pretreated, $45 \mathrm{~min}$ earlier, with either the $\alpha$-adrenergic antagonist prazosin $(5 \mathrm{mg} / \mathrm{kg}$, i.p.) or the serotonergic antagonist methysergide $(0.5$ $\mathrm{mg} / \mathrm{kg}$, i.p.). These doses of prazosin and methysergide were chosen since, in the preceding paper, we found that they specifically blocked the increase in the masseteric reflex produced by infusions of NE and serotonin (5-HT), respectively, into MoV.

In the second series, a separate group of cats, previously prepared with bilateral injection cannulae in the MoV (coordinates $\mathrm{P}-4.5, \mathrm{~L}$ $+3.7, \mathrm{~V}-5.0)$ as described in the preceding paper, were exposed to the dog, white noise, and click and the reflex response elicited and measured bilaterally. Local infusions of 6-hydroxydopamine hydrochloride (6-OHDA) were then made unilaterally into $\mathrm{MoV}$ in order to produce specific destruction of NE terminals. One microliter of a $4 \mu \mathrm{g} / \mu \mathrm{l}$ solution of 6-OHDA (calculated as the free base) was infused over a 2-min interval; during the same session, $1 \mu \mathrm{l}$ of the vehicle $(0.2 \mathrm{mg} / \mathrm{ml}$ ascorbic acid in $0.9 \%$ physiological saline) was infused into the contralateral side. All infusions were made while the cat was awake and unrestrained. One week later, cats were re-exposed in a random order to the aforementioned conditions, and the reflex response again elicited and measured bilaterally. In addition, cats were tested with a low dose of NE $(0.063$ $\mu \mathrm{g}$ in 1 subject; 0.125 in the remaining 4$)$ and $5-\mathrm{HT}(0.125 \mu \mathrm{g}$ in all 5 subjects) before and after the lesion. The doses used were the lowest ones found to produce a suprathreshold response in these same animals. This allowed us to evaluate the efficacy and specificity of the denervation pharmacologically by testing for an enhanced response only to NE, suggesting catecholamine denervation supersensitivity.

Assessment of neurotoxic lesions. At the end of the experimental procedure, animals were deeply anesthetized with sodium pentobarbitol $(50 \mathrm{mg} / \mathrm{kg}$, i.p.) and their brains were exposed by removing the overlying portions of the calvarium. They were then overdosed with additional pentobarbitol $(50 \mathrm{mg} / \mathrm{kg}$, i.v.) and their brains rapidly removed and placed into liquid nitrogen. The frozen brains were blocked and a piece of brain-stem tissue was placed in a microtome and sectioned until MoV was reached. A length of sharpened 13-gauge stainless steel tubing was used to punch the center of the nuclei to be assayed. In most cases, it was possible to follow the tracks of the injection cannulae to their final position in $\mathrm{MoV}$ and use this to guide the bilateral punch locations. If no cannulae tracks were visible, stereotaxic atlas landmarks were used and bilateral punches were taken $4 \mathrm{~mm}$ lateral to the midline, rostral to the facial genu. Similar-sized punches of areas immediately medial and ventral to MoV were also taken as control tissue in order to determine specificity of the lesion.

The levels of NE and 5-HT were assayed using high-performance 


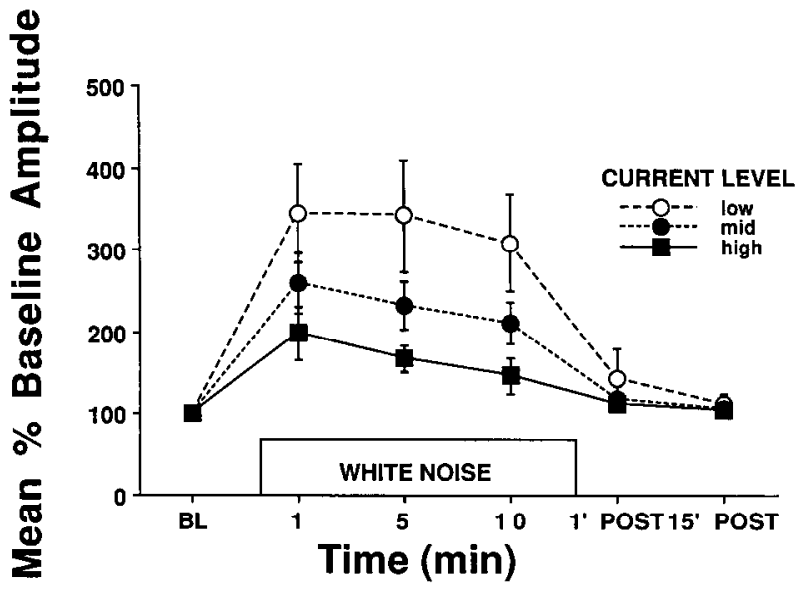

Figure 1. Effects of $15 \mathrm{~min}$ exposure to $100-\mathrm{dB}$ white noise on the masseteric reflex response seen at low (open circles), middle (closed circles), or high (closed squares) current intensitics. Valucs arc mean percent of baseline $(B L)$, which was $35.8 \mu \mathrm{V}( \pm 3.35 \mathrm{SEM})$ at the low current, $85.7 \pm 3.77$ at the middle, and $175.1 \pm 17.9$ at the high current. Responses during min 1,5 , and 10 of white noise are significantly different from BL and from both the 1 - and 15 -min post-white noise values at all current levels $(p<0.01$, Newman-Keuls).

liquid chromatography (HPLC) with electrochemical detection. Samples were prepared by homogenizing the tissue in ice-cold perchloric acid solution containing sodium metabisulfite (an antioxidant). Homogenates were centrifuged at $15,000 \mathrm{~g}$ for $7 \mathrm{~min}$ and $30 \mu \mathrm{l}$ of the resulting supernatants injected, using a 100- $\mu$ l Hamilton microsyringe, directly into an Bioanalytical Systems, Inc., HPLC system. The peak heights were measured and compared to peak heights of a standard solution containing known amounts of NE and 5-HT. Sample values were determined by the ratio of unknown to standard peak heights and expressed as $\mathrm{ng} / \mathrm{mg}$ of wet tissue.

Drugs. Drugs used were NE hydrochloride (Sigma); 5-hydroxytryptamine creatine sulfate (Sigma); 6-OHDA (Sigma); methysergide maleate (Sandoz); prazosin hydrochloride (Pfizer). NE, methysergide, and 5-HT were dissolved in physiological saline; prazosin was dissolved in heated propylene glycol; 6-hydroxydopamine was dissolved in physiological saline containing $0.2 \mathrm{mg} / \mathrm{ml}$ ascorbic acid and kept on ice. Doses are expressed as the salt, except for 6-hydroxydopamine, which is calculated as the free base. Solutions were made fresh just prior to each session.

Data analysis. Effects of white noise, dog, and click were first analyzed using a repeated-measures analysis of variance (ANOVA); post hoc comparisons between individual time points were then analyzed with Newman-Keuls tests. For the denervation studies, the ratio between the post- and the prelesion response to white noise and dog was calculated for each subject on both the lesion and the control sides. These scores were then entered into a within-subject ANOVA. It was not possible to obtain bilateral pre- and postlesion readings for all subjects in the click condition; therefore, a within-subject ANOVA was performed comparing postlesion measurements on the lesioned side versus the control side.

\section{Results}

\section{Environmental manipulations}

White noise. The effect of $15 \mathrm{~min}$ exposure to $100-\mathrm{dB}$ white noise on the amplitude of the masseteric reflex response was examined in 7 cats. The typical behavioral response to the white noise was a flattening of the ears and assumption of a crouched position, with little overt motor activity. As shown in Figure 1, the amplitude of the masseteric reflex was significantly increased, at all current levels, above baseline levels during the white noise exposure $[F(5,30)=12.3, p<0.001]$. The results of a Newman-Keuls test revealed that compared to prenoise baseline, the reflex amplitude was significantly elevated at min

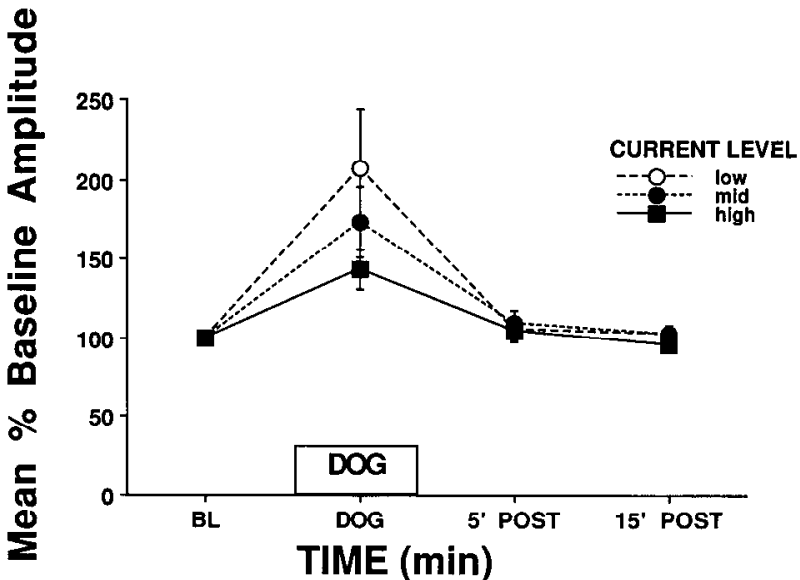

Figure 2. Effects of a 5-min visual confrontation with a dog on the masseteric reflex response seen at the low (open circles), middle (closed circles), or high (closed squares) current intensities. Values are mean percent of baseline $(B L)$, which was $67.1 \mu \mathrm{V}( \pm 26.5$ SEM) at the low current, $148.4 \pm 35.3$ at the middle, and $255.8 \pm 47.1$ at the high current. Response during the dog exposure is significantly different from all other time points at all current levels ( $p<0.01$, Newman-Keuls).

1,5 , and 10. The magnitude of the increase was greatest at the low current level (344.7\% of baseline during min 1 of white noise), but it was still substantial at the middle $(259.3 \%$ of baseline) and high ( $198.9 \%$ of baseline) currents. The amplitude remained elevated for the duration of the noise, showing some evidence of adaptation or habituation. Upon termination of white noisc, the amplitude quickly returncd to baseline levels. When tested starting $1 \mathrm{~min}$ post-white noise, the response amplitude was $142.6 \%$ of baseline at the low current, $118 \%$ at the middle current, and $112 \%$ at the high current. By 15 min postwhite noise, the amplitude was $111 \%$ of baseline at the low, $105.9 \%$ of baseline at the middle, and $104.2 \%$ of baseline at the high current level.

Dog. The amplitude of the masseteric reflex was examined in 6 cats during a 5-min exposure to a dog. Most subjects responded to the presence of the dog with a stereotyped feline defense reaction: arched back, piloerection, hissing, and a broadside stance. As can be seen in Figure 2, the presence of a dog significantly increased the amplitude of the masseteric reflex response $[F(3,15)=8.18, p<0.001]$. The increase was evident at all current levels, increasing to $207 \%$ of baseline at the low current, $173.5 \%$ of baseline at the middle current, and $143.6 \%$ of baseline at the high current. As with the white noise, the amplitude quickly returned to baseline upon removal of the dog. Five minutes after the exposure, the reflex amplitude did not differ from baseline values $(102.7 \%, 109.1 \%$, and $104.3 \%$ of baseline at the low, middle, and high current levels, respectively).

Click. The effect of clicks presented at various intervals before MesV stimulation was tested in 11 cats (Fig. 3). Clicks presented 100 and $150 \mathrm{msec}$ prior to stimulation significantly augmented the masseteric reflex by $43.9 \%$ and $37.1 \%$, respectively. Interestingly, this increase is considerably less than that observed in response to the other 2 conditions, white noise and dog, which produced a 2-6-fold increase in reflex response amplitude at the low current level.

In contrast to the facilitation observed at the 100- and 150msec intervals, clicks presented $20 \mathrm{msec}$ prior to stimulation significantly decreased the amplitude of the masseteric reflex to 


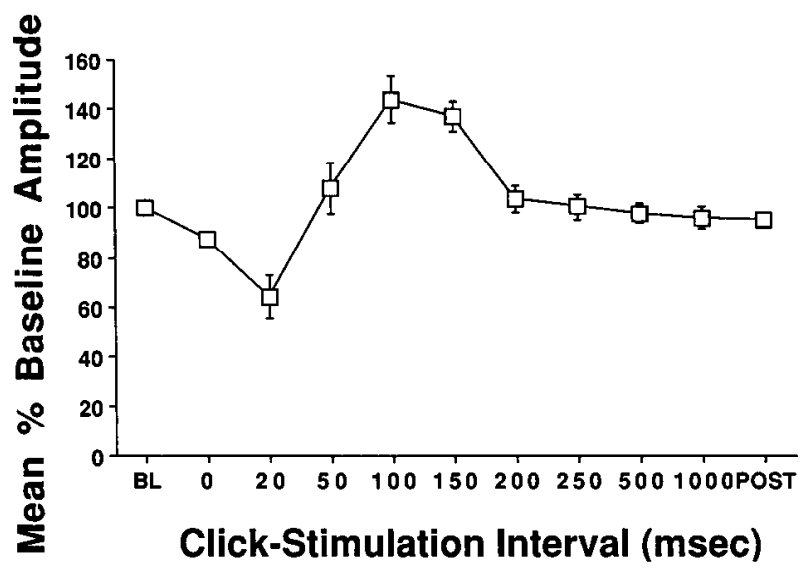

Figure 3. Effects on the masseteric reflex response of clicks presented $0,20,50,100,150,200,250,500$, or 1000 msec prior to MesV stimulation. Compared to preclick baseline $(B L)$, which was $47.75 \pm 2.1$ $\mu \mathrm{V}$, the response was significantly inhibited by clicks presented $20 \mathrm{msec}$ prior to stimulation and significantly enhanced by clicks presented 100 and $150 \mathrm{msec}$ prior to stimulation ( $p<0.01$, Newman-Keuls). At all other time points, the reflex amplitude was not significantly different from $B L$.

$64.3 \%$ of baseline $[F(10,20)=21.8, p<0.001]$. Clicks presented at all other intervals tested had no effect on the reflex amplitude.

\section{Antagunist tests}

White noise. The effect of $100-\mathrm{dB}$ white noise was tested at the low current level in 2 groups of 5 cats each: one pretreated with the $\alpha$-adrenergic antagonist prazosin $(5 \mathrm{mg} / \mathrm{kg}$, i.p.) and the other pretreated with the serotonergic antagonist methysergide $(0.5$ $\mathrm{mg} / \mathrm{kg}$, i.p.). As in the preceding paper, administration of both prazosin and methysergide decreased the baseline amplitude of the masseteric reflex by approximately $40 \%$ below predrug baseline levels, suggesting that $\mathrm{NE}$ and $5-\mathrm{HT}$ may provide a tonic facilitatory influence. There was, however, no dramatic druginduced state change following systemic administration of either antagonist; careful observation of the subjects indicated that they were awake and responsive to external stimuli. Figure 4 depicts the subsequent response to white noise in both groups. In the prazosin-pretreated subjects, white noise presentation had no effect on the amplitude of the masseteric reflex $[F(5,20)=$ $0.94, p>0.05$ ]. The amplitude was $102 \%$ of baseline at min 1 of white noise, $93.6 \%$ of baseline during min 5 , and $109 \%$ during $\min 10$.

In methysergide-pretreated subjects, by contrast, the response to white noise was similar to that described previously in untreated subjects. Following methysergide pretreatment, the amplitude of the reflex response was significantly increased during white noise presentation to $197 \%$ of baseline during $\min 1,166 \%$ during $\min 5$, and $173 \%$ during $\min 10[F(5,20)=25.5, p<$ $0.001]$. The response returned to baseline levels by $1 \mathrm{~min}$ after termination of the white noise.

Click. Figure 5 compares the masseteric reflex response to click following methysergide $(n=5)$ or prazosin $(n=7)$. In both groups, clicks presented $20 \mathrm{msec}$ prior to stimulation significantly suppress the masseteric reflex response, as described above for the nondrug conditions. In the prazosin group, all other clickstimulation intervals are ineffective in altering the reflex response, whereas in methysergide-treated subjects, the amplitude of the masseteric reflex is significantly enhanced by clicks pre-

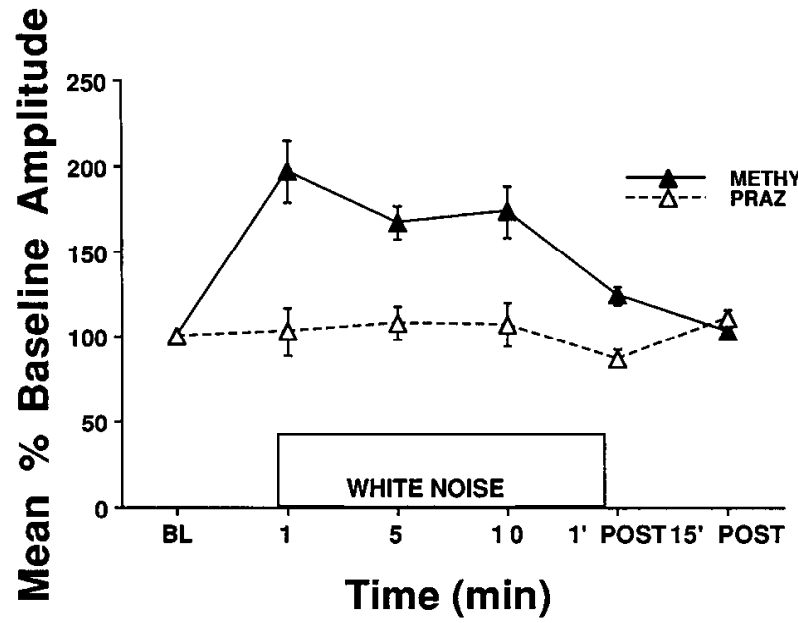

Figure 4. Effects of $15 \mathrm{~min}$ of $100-\mathrm{dB}$ white noise on the masseteric reflex in groups pretreated $45 \mathrm{~min}$ earlier with either prazosin $(P R A Z$; $5 \mathrm{mg} / \mathrm{kg}$, i.p., open triangles) or methysergide (METHY;0.5 mg/kg, i.p., closed triangles). Baseline $(B L)$ values were $40.1( \pm 3.44$ SEM) for the PRAZ group and 47.2 $( \pm 6.67$ SEM) for the METHY group. Response during $\min 1,5$, and 10 of white noise in the METHY group was significantly different from both BL and post-white noise values $(p<$ 0.01 , Newman-Keuls). There was no significant effect of white noise on the amplitude of the reflex response in the PRAZ group.

sented at 100 (127.6\% of baseline) and 150 (130.3\% of baseline) msec prior to stimulation $[F(10,40)=6.03, p<0.001]$.

\section{Denervation studies}

Neurochemical assay. Infusions of the catecholamine neurotoxin 6-OHDA into MoV reduced the NE content to a mean of $32.4 \%$ of the vehicle-injected contralateral control. Levels of 5 -HT were not affected ( $140 \%$ of control), indicating that the destruction was indeed specific for NE. Moreover, NE levels in punches from control sites adjacent to MoV did not differ between the denervated versus the control sides, showing that the NE depletion was restricted to MoV. Finally, the baseline amplitude remaincd unchanged on both sides following denervation procedures.

White noise. On the first exposure, 100-dB white noise increased the amplitude of the masseteric reflex on both the tobe-lesioned $(655.2 \%$ of baseline during min 1, 370.1\% during $\min 5$, and $292.2 \%$ during min 10$)$ and control sides $(312.3 \%$ of baseline during min $1,286.1 \%$ during $\min 5$, and $253.7 \%$ during min 10), as described above for untreated subjects. When the response to white noise was retested 7-9 days following unilateral 6-OHDA infusion, the masseteric reflex response on the denervated side was markedly attenuated (increasing to only $170.8 \%$ of baseline during $\min 1,164.9 \%$ during $\min 5$, and $123.6 \%$ during min 10; Fig. 6, top). However, the amplitude of the reflex response on the control side was increased to approximately the same degree as before $(258 \%$ of baseline during $\min 1,239.7 \%$ during $\min 5$, and $195.6 \%$ during min 10; Fig. 6 , bottom), precluding an cxplanation bascd simply on habituation to the stimulus. Analysis comparing the difference in response pattern between the lesioned and control sides revealed a significant condition $\times$ time interaction $[F(5,20)=4.4, p<$ $0.01]$, indicating that the response during white noise on the lesioned side was significantly lower than that on the control side.

Dog. Similarly, augmentation of the reflex response by con- 


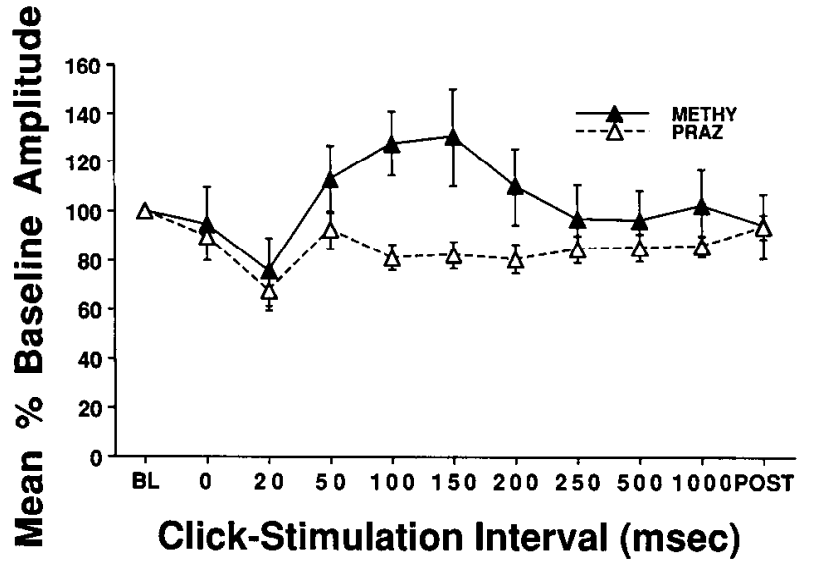

Figure 5. Effects on the masseteric reflex response of clicks presented at various intervals prior to MesV stimulation in groups pretreated with either prazosin (PRAZ; $5 \mathrm{mg} / \mathrm{kg}$, i.p., open triangles) or with methysergide (METHY;0.5 mg/kg, i.p., closed triangles). When compared to the appropriate baseline $(B L)$ values, which were $41.6 \pm 3.1 \mu \mathrm{V}$ for the PRAZgroup and 43.6 $\pm 2.03 \mu \mathrm{V}$ for the METHY group, clicks presented $20 \mathrm{msec}$ prior to stimulation significantly suppressed the reflex response in both PRAZ and METHY groups; clicks presented 100 and $150 \mathrm{msec}$ prior to stimulation significantly enhanced the reflex response in the METHY group ( $p<0.01$, Newman-Keuls). In the PRAZ group, all other click-stimulation intervals had no effect on the reflex response.

frontation with a dog was significantly reduced on the lesioned side when compared to the response on the control side $[F(1,3)$ $=10.63 ; p<0.003 ;$ Fig. 7$)$. During the first exposure to a dog, the reflex amplitude increased to $289 \%$ of baseline on the tobe-lesioned side and to $159 \%$ of baseline on the control side. Following denervation, dog presentation increased the reflex to only $130 \%$ of baseline on the lesioned side and to $235 \%$ of baseline on the control side. Again, the fact that no decrement in response to dog was observed on the control side (in fact, a slight increase was seen) argues against habituation.

Click. Denervation also significantly affected the masseteric reflex response to click $[F(1,4)=7.43, p<0.05]$. When tested on the control side, clicks presented at 100 and $150 \mathrm{msec}$ prior to stimulation significantly enhanced the amplitude of the reflex response by $31 \%$ and $37 \%$, respectively; clicks presented $20 \mathrm{msec}$ prior to stimulation suppressed the amplitude of the reflex response by $51 \%$ (Fig. 8). When tested on the lesioned side, clicks presented at 100 and $150 \mathrm{msec}$ prior to stimulation had no effect on the reflex response; however, a marked suppression was still produced by clicks presented $20 \mathrm{msec}$ prior to stimulation (Fig. 8 ), indicating that the denervation effect is not simply attributable to nonspecific damage. In addition, this finding implies that the inhibition produced by clicks presented $20 \mathrm{msec}$ prior to MesV stimulation is not mediated by noradrenergic mechanisms. Data from an individual subject tested bilaterally to the click before and after denervation are presented in Figure 9. Note that the baseline amplitudes for the control and lesion sides do not differ at any point. During the first click test, both sides exhibit a similar response. When retested, the denervated side does not exhibit the previously observed increase (Fig. 9, top), whereas the control side does (Fig. 9, bottom) indicating that the decrement observed on the lesioned side during the second test cannot be attributed to habituation.

Pharmacological tests. Figure 10 compares the response to
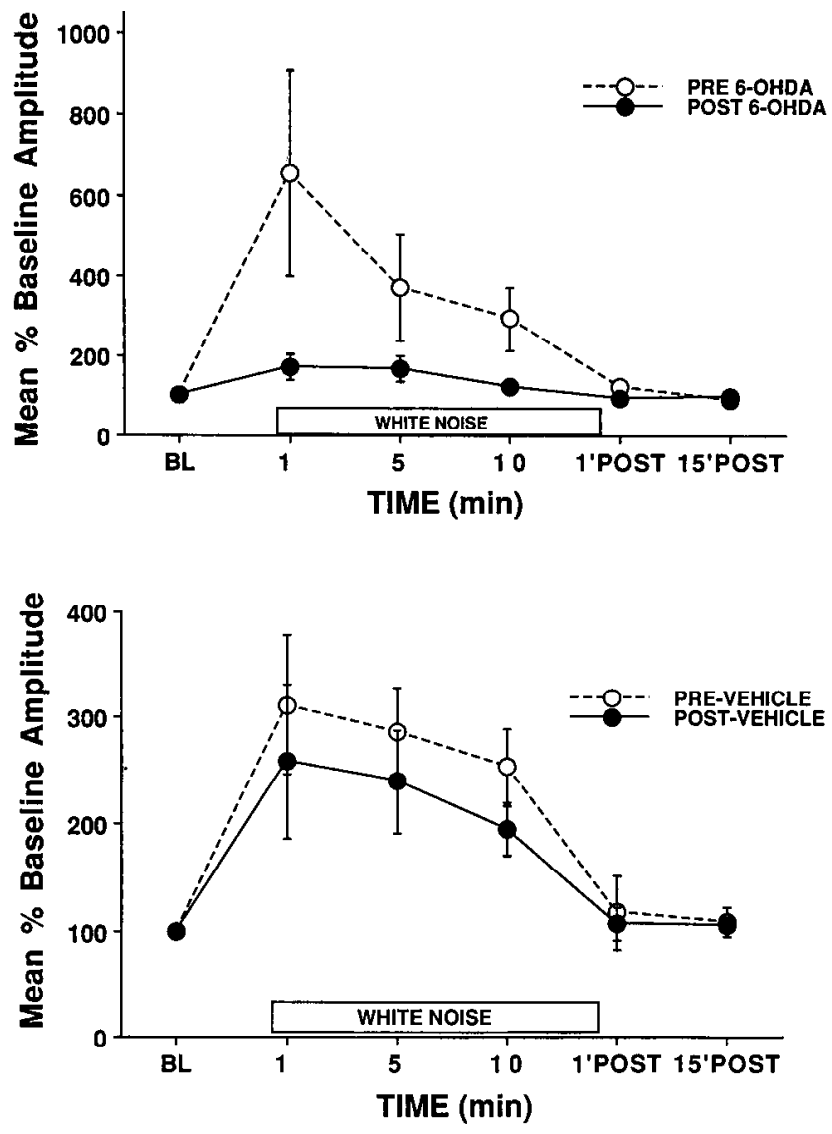

Figure 6. Top, Effects of $15 \mathrm{~min}$ of $100-\mathrm{dB}$ white noise on the masseteric reflex response before (open circles) and 1 week after (closed circles) local infusion of 6-OHDA in MoV. White noise significantly increased the reflex response during min 1,5 , and 10 when tested prior to 6-OHDA ( $p<0.01$, Newman-Keuls), but had no effect when tested after 6-OHDA. Bottom, Effects of $15 \mathrm{~min}$ of $100-\mathrm{dB}$ white noise on the masseteric reflex response before (open circles) and 1 week after (closed circles) local infusion of vehicle $(0.2 \mathrm{mg} / \mathrm{ml}$ ascorbic acid in $0.9 \%$ physiological saline) in MoV. White noise significantly increased the reflex response during min 1,5 , and 10 when tested both prior to and 1 week following vehicle infusion ( $p<0.01$, Ncwman-Kculs).

microinfusion of NE (top) or 5-HT (bottom) tested, in the same subjects, before and after 6-OHDA. During the initial test, infusion of both NE ( $0.125 \mu \mathrm{g}$ in 4 subjects, $0.063 \mu \mathrm{g}$ in 1 subject) and 5 -HT $(0.125 \mu \mathrm{g}$ in all 5 subjects $)$ produced a moderate increase in the masseteric reflex response. NE increased the reflex response from a baseline of $58.2 \mu \mathrm{V}$ to $108 \mu \mathrm{V}$ in min 1 and to $89 \mu \mathrm{V}$ in min 5 ; by $\min 15$, the response returned to baseline levels. Similarly, 5-HT infusion increased the response from a $56.3-\mu \mathrm{V}$ baseline to $77.9 \mu \mathrm{V}$ in min 1 and to $80.7 \mu \mathrm{V}$ in min 5. One week after administration of 6-OHDA, the same doses were retested and, as noted above, baselines were unchanged. The response to NE was significantly greater and longer-lasting than the predenervation response $[F(1,4)=13.1, p$ $<0.02]$. On the second test, NE increased the amplitude of the masseteric reflex from a baseline amplitude of $56.7 \mu \mathrm{V}$ to 161.6 $\mu \mathrm{V}$ in $\min 1,322.2 \mu \mathrm{V}$ in $\min 5,166.7 \mu \mathrm{V}$ in $\min 15$, and 105.6 $\mu \mathrm{V}$ in $\min 30$. When 5-HT was retested following denervation, the reflex amplitude did not significantly differ from the previous test response. These findings suggest that the enhancement observed to NE reflects receptor supersensitivity caused by specific destruction of the NE terminals in MoV. 

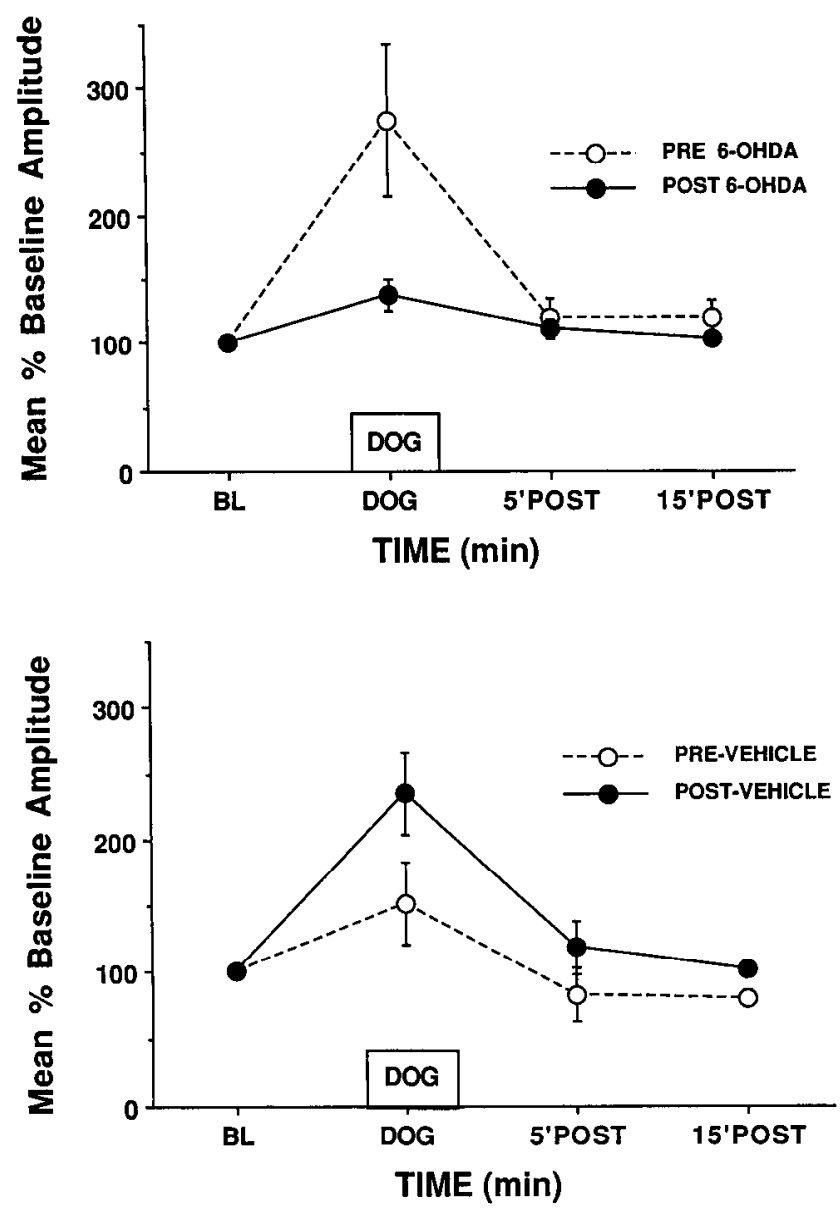

Figure 7. Top, Effects of a 5-min confrontation with a dog on the masseteric reflex response before (open circles) and 1 week after (closed circles) ipsilateral local infusion of 6-OHDA in MoV. Dog exposure significantly increased the reflex response when tested prior to 6-OHDA $(p<0.01$, Newman-Keuls), but not when tested after 6-OHDA. Bottom, Effects of a 5-min confrontation with a dog on the masseteric reflex response before (open circles) and 1 week after (closed circles) local infusion of vehicle $(0.2 \mathrm{mg} / \mathrm{ml}$ ascorbic acid in $0.9 \%$ physiological saline) in the ipsilateral MoV. Dog exposure significantly increased the reflex response when tested both prior to and 1 week following vehicle infusion $(p<0.01$, Newman-Keuls).

\section{Discussion}

In the present series of studies, conditions known to activate NE neurons in cats (exposure to 100-dB white noise, confrontation with a dog, and presentation of clicks) were also shown to increase the amplitude of the masseteric reflex response. This suggests that NE release, under physiological conditions, facilitates the masseteric reflex, as was demonstrated in the preceding paper for pharmacologically induced increases in NE neurotransmission.

In the previous paper, the increase in amplitude was a function of NE dose. Here we find that the magnitude of the increase is dependent on the type of stimulus employed; tonic stimuli (such as white noise or dog) result in a much greater percent facilitation than do phasic stimuli (such as clicks). This may indicate that tonic stimuli per se release more endogenous NE or, alternatively, that the effect reflects the relative strength of the stimuli (i.e., that white noise and dog are stronger activators of $\mathrm{NE}$ neurons than are clicks). Consistent with this interpretation,

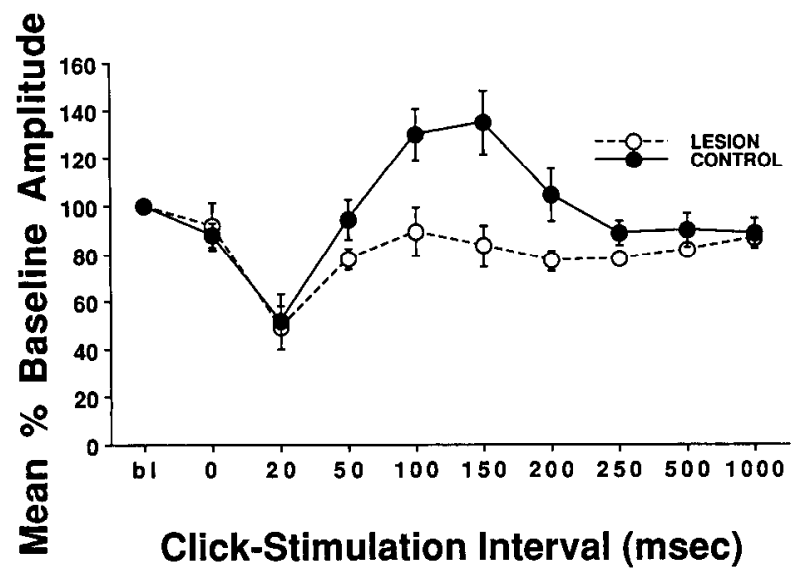

Figure 8. Effects on the masseteric reflex of clicks presented at various intervals prior to MesV stimulation following infusion of 6-OHDA (open circles) into $1 \mathrm{MoV}$ or vehicle control $(0.2 \mathrm{mg} / \mathrm{ml}$ ascorbic acid in $0.9 \%$ physiological saline, closed circles) in the other MoV. When compared to the appropriate baseline $(B L)$ values, which were $51.8 \pm$ $3.06 \mu \mathrm{V}$ for the LESION side and 52.6 $\pm 2.65 \mu \mathrm{V}$ for the CONTROL side, clicks presented $20 \mathrm{msec}$ prior to stimulation significantly suppressed the reflex response in both LESION and CONTROL groups; in addition, clicks presented 100 and $150 \mathrm{msec}$ prior to stimulation significantly enhanced the reflex response in the CONTROL group ( $p$ $<0.01$, Newman-Keuls). In the LESION group, all other click-stimulation intervals had no effect on the reflex response.

white noise and dog have been shown to produce a 2-3-fold increase in the activity of LC-NE neurons (Abercrombie and Jacobs, 1987; Levine ct al., 1987) whercas clicks produce a short-lasting activation, typically of 1-3 spikes (Rasmussen et al., 1986).

The time course of the reflex facilitation parallels the known time course and pattern of neuronal activation produced by the stimuli. Neurophysiological studies of cat LC-NE neurons show that unit activity is increased tonically by exposure to $15 \mathrm{~min}$ of white noise and during $5 \mathrm{~min}$ exposure to a dog (Abercrombie and Jacobs, 1987; Levine et al., 1987). The increase in unit activity during white noise is greatest during $\min 1$ and then shows a slight adaptation in min 5 and 10; the same pattern was observed for the increase in the masseteric reflex response during white noise. In addition, both unit activity and the amplitude of the reflex response return to baseline levels shortly after stimulus offset. LC neurons are also activated phasically in response to repetitive presentation of clicks, with a latency of $50 \mathrm{msec}$ and duration of $100 \mathrm{msec}$ (Rasmussen et al., 1986). Consistent with this, the present results show that clicks produce reflex facilitation only when presented 100 or 150 msec prior to MesV stimulation, which corresponds precisely to the period during which NE neurons are activated by the same stimulus.

While the source of the NE innervation of MoV in cat is still undetermined, anatomical studies in rat indicate that the $\mathrm{NE}$ input is provided by neurons in the lateral tegmental (A7) NE group, and not by LC neurons (Vornov and Sutin, 1983; Grzanna et al., 1987). There is evidence, however, that the activity of cat A7 NE neurons is similar to that of cat LC-NE neurons. The activity of A7 neurons has been shown to be state-dependent, with discharge rate highest during periods of active waking and decreasing during periods of drowsiness and slow-wave sleep; moreover, A7 neurons show virtually a complete cessation of activity during REM sleep (Reiner, 1985, 1986). In addition, pilot studies from our laboratory indicate that $A 7$ neu- 

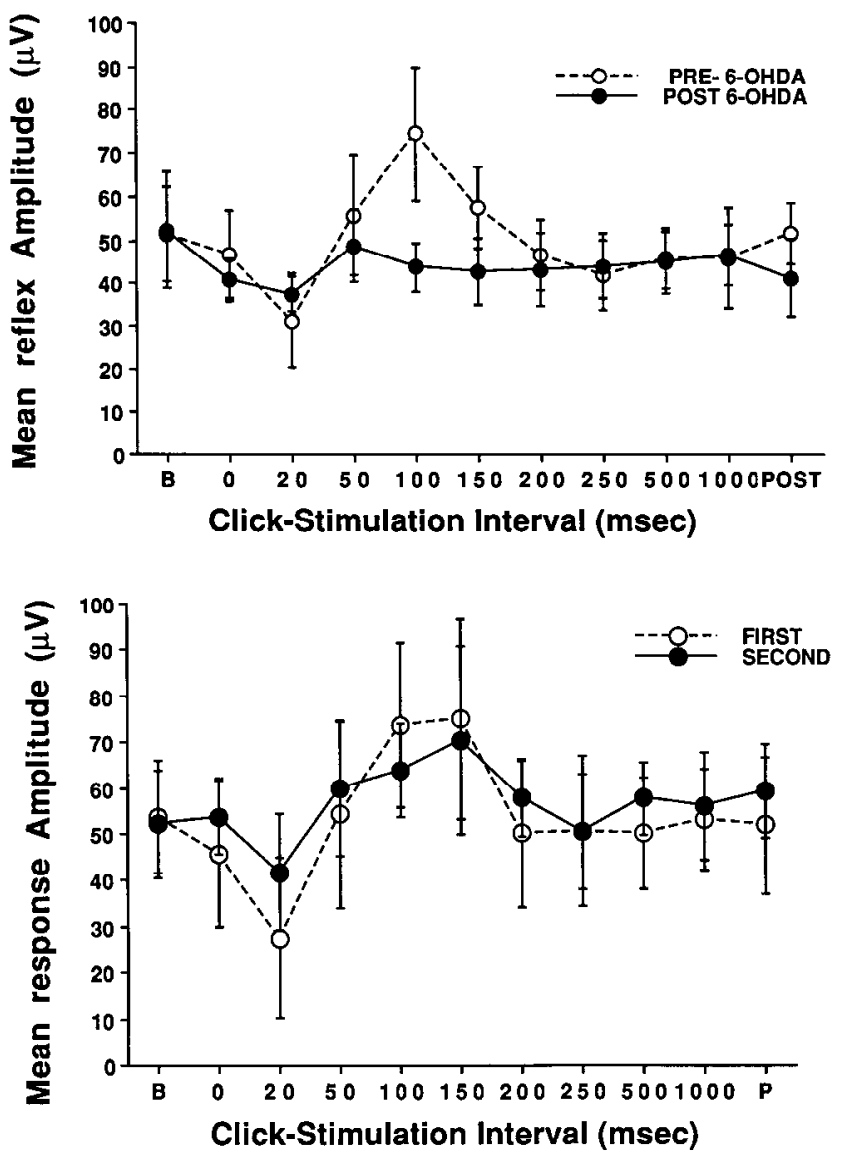

Figure 9. Top, Representative data from an individual subject showing the response to clicks before (open circles) and after (closed circles) infusion of 6-OHDA. Bottom, Representative data from the same subject showing the contralateral masseteric reflex response to clicks before (open circles) and after (closed circles) infusion of vehicle.

rons are activated by exposure to white noise and clicks, as are LC neurons. In summary, the results of the first series of studies show that conditions known to activate NE neurons are correlated with increases in the masseteric reflex and suggest that release of NE is responsible for the augmentation.

In the next series of studies, blockade of NE neurotransmission by pretreatment with the $\alpha$-1-adrenergic antagonist prazosin blocked the reflex augmentation in response to white noise or click. This result is consistent with the hypothesis that the augmentation of the reflex response produced by white noise and click reflects increased NE synaptic activity and suggests that the augmentation is mediated by $\alpha-1$ receptors, although a role for other receptors cannot be ruled out. The response to these stimuli was not, however, affected by pretreatment with the 5-HT antagonist methysergide, at a dose shown in the preceding paper to block the increase in the reflex response produced by local infusion of $5-\mathrm{HT}$, thus providing evidence for at least some neurochemical specificity of the blockade.

Every attempt was made to keep behavioral state consistent across experimental and control conditions through the use of behavioral and polygraphic criteria. Nonetheless, it is possible that the effects of systemic administration of prazosin and methysergide could be attributable to alterations in state. Several lines of evidence, however, make this unlikely. First, the same results seen with systemic prazosin were observed following unilateral destruction of NE nerve terminals in MoV. Both ma-
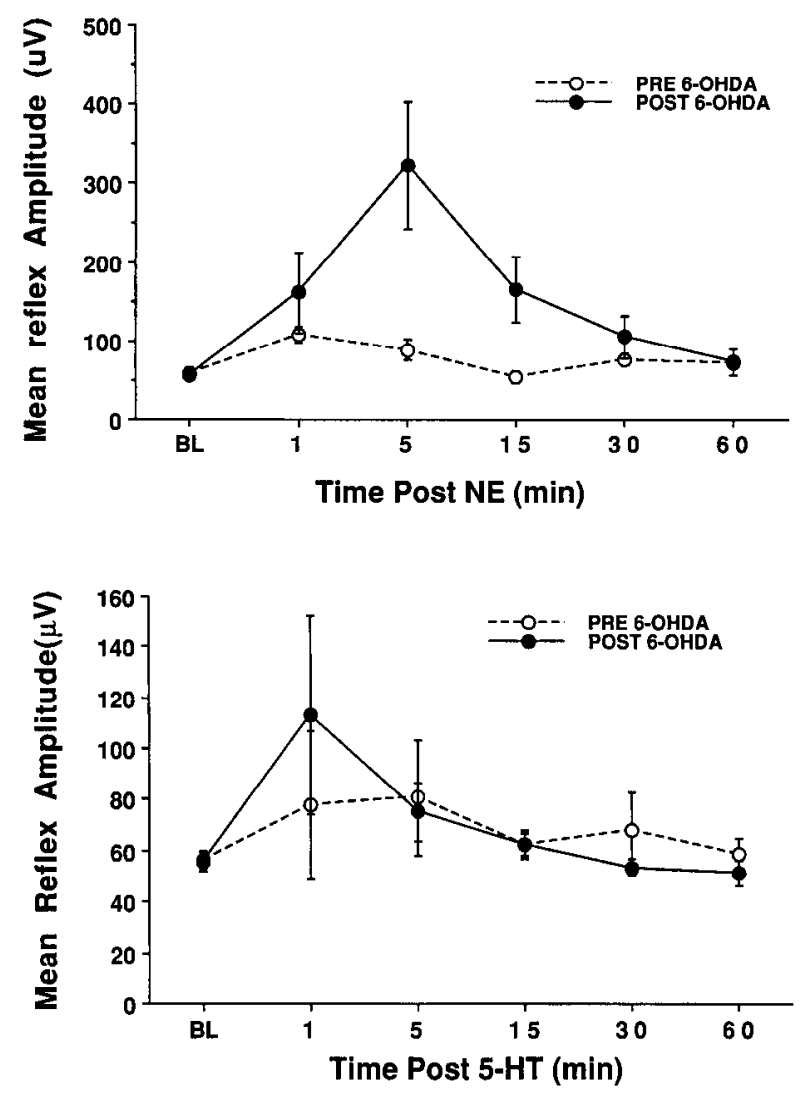

Figure 10. Top, Group data showing the response to infusions of low doses of NE before (open circles) and after (closed circles) infusion of 6-OHDA. Bottom, Group data from the same subjects as above showing the response to infusions of low doses of 5-HT beforc (open circles) and after (closed circles) infusion of 6-OHDA.

nipulations produced a virtually complete blockade of the augmenting effects on the reflex of clicks and white noise. As an important control, the reflex response on the nondenervated side displayed the typical augmentation. These identical results provide strong confirmation that the prazosin-induced antagonism of increases in the masseteric reflex in response to environmental manipulations reflects a blockade of NE activity and is not caused by a global behavioral state change. Second, while the amplitude of the masseteric reflex is indeed largest during periods of active waking and smallest during REM sleep, there is little or no difference in amplitude in quiet waking as compared to slow-wave sleep (Chase et al., 1968). Since all tests were conducted while the subjects were unambiguously awake, any small drug-induced state change would not be expected to so dramatically affect the reflex amplitude. Thus, it is worth noting that the prazosin pretreatment almost completely antagonized the large facilitatory effects of local NE infusion on the masseteric reflex response (preceding paper) and white noise (this paper). Such gross alterations in the reflex cannot, therefore, be attributed to subtle drug-induced state changes.

The almost complete loss of reflex facilitation produced by environmental stimuli following blockade of NE transmission suggests that the increases in motor behavior produced by various activators or stressors may be attributable almost completely to NE. The generality of this finding to other motor systems remains, however, to be determined. It is also possible that ncurochemical systems other than NE, such as 5-HT, may 
be importantly involved in such effects but that NE is necessary for their expression.

Evidence for a causal relationship between activation of $\mathrm{NE}$ neurons and increases in the masseteric reflex was provided by the last series of experiments. Specific unilateral destruction of the NE terminals in MoV following local infusion of 6-OHDA blocked or significantly attenuated, only on the denervated side, the augmentation of the reflex response previously produced by white noise, dog, and click. The response on the control, vehicleinjected, side was unaffected, thus ruling out habituation as an explanation for these results. As noted in the preceding paper, both methysergide and prazosin decreased the baseline amplitude of the reflex response, suggesting that 5-HT and NE inputs may provide tonic facilitation. If so, one would expect to also see a decrease in baseline following removal of NE terminals in MoV by 6-OIIDA. No such decrease was noted, however, suggesting that tonic NE release may be sufficient, but not necessary. For example, tonic 5-HT release, presumably unaffected by 6-OHDA, may maintain the level of excitability of MoV neurons. In addition, the observed supersensitivity to NE may compensate for smaller endogenous levels of $\mathrm{NE}$.

These data provide compelling evidence that the facilitation of the reflex produced by the aforementioned conditions is mediated by release of NE from axon terminals in MoV. Moreover, they provide the first demonstration that endogenous release of NE, at a specific site and under physiological conditions, facilitates behavioral output in the intact, behaving animal. Thus, the results of the present experiments, in conjunction with those described in the preceding paper, validate the use of the masseteric reflex as a model system to examine the functional significance of changes in NE neurotransmission in the CNS of behaving animals.

A number of interesting questions about the mechanism of action of NE can be addressed by our results. The modulatory effects mediated by $\alpha-1$-adrenoceptors in response to iontophoretic application of NE have typically been characterized as long-lasting, exhibiting a time course of seconds to minutes and generally outlasting the NE application (Aghajanian and Rogawski, 1983). Our results, however, indicate that the time course of NE modulation under physiological conditions may be much more discrete, lasting for only the period during which NE neurons are activated. Within the first minute following termination of white noise or removal of the dog, the masseteric reflex response amplitude returned to baseline levels. In addition, the facilitation produced by a single click was precisely time-locked to the period of NE activation produced by that click. These data indicate that the NE released by the brief activation (1-3 spikes) of NE neurons lasts for no longer than 20-50 msec after termination of neuronal activity. Thus, it appears that the modulatory effects of physiologically released NE are short-lived, unlike that reported in iontophoretic studies. This may be due to the iontophoretically released NE overwhelming the uptake mechanism which normally rapidly removes the neurotransmitter from the synaptic cleft. Another possibility is that iontophoretically released NE activates a second messenger system that is not engaged under the physiological conditions employed here. Indeed, the facilitation of the masseteric reflex in response to infusion of NE we describe in the preceding paper displays a much longer time course than that observed in response to the environmental manipulations used here.

In addition to NE neurotransmission, the masseteric reflex can be a useful tool in studying a number of other issues. U1- trastructural studies document the existence of 4 morphologically distinct classes of axon terminals synapsing on MoV neurons, characterized by either round or flat vesicles within lucent or dense cytoplasm and showing differential distribution on various portions of the soma and dendritic arbor (Card et al., 1986). As the presence of round or flat vesicles within boutons has been linked with excitatory and inhibitory inputs (Uchizono, 1965), this raises the possibility of examining different types of neurotransmission using the masseteric reflex as a model system. For example, an interesting question would be to characterize inhibitory influences on the masseteric reflex. As described above, clicks presented $20 \mathrm{msec}$ prior to MesV stimulation significantly decrease the amplitude of the reflex response. The inhibition we observed is similar to that reported by Wright and Barnes (1972). Among other patterns, they reported that the amplitude of the lumbar monosynaptic reflex in anesthetized or decerebrate cats was inhibited by tone bursts presented 20-30 msec prior to dorsal root stimulation. Lesions of the medial pons and medulla, including the medial reticular formation, selectively abolished this inhibition. In addition, the amplitude of the startle reflex has also been shown to be inhibited by the presentation of an auditory (or visual) stimulus prior to the startle-eliciting stimulus, although the optimal interstimulus interval in this paradigm is between 50 and $150 \mathrm{msec}(\mathrm{Wu}$ et al., 1988). The neural mechanisms underlying this prepulse inhibition are not clear. Some evidence indicates that it may be cholinergically mediated (Leitner et al., 1981), suggesting that the click-induced inhibition of the masseteric reflex may also reflect cholinergic mechanisms. Indeed, our results show that the click-induced suppression is not prevented by blockade of either NE or serotonergic transmission, implying the influence of another system. In further support of this hypothesis, anatomical studies reveal that cholinergic input into MoV may be provided by extensive bilateral projections from cells in the supratrigeminal and intertrigeminal nuclei (Travers and Norgren, 1983; Vornov and Sutin, 1983), an area where muscarinic cholinergic receptors have been localized by immunohistochemistry (Wamsley et al., 1981). Finally, neurophysiological studies suggested that cells in the supratrigeminal area inhibit masseteric motoneurons during jaw opening (Kidokoro et al., 1968).

Other areas of research that could be studied using the masseteric reflex response as a model system include neurotransmitter interactions (e.g., between NE and 5-HT systems); functional consequences of co-localization of neurotransmitters (Substance P has been co-localized in 5-HT terminals in MoV); and neurotransmitter-hormonal interactions. We are currently attempting to determine whether an opposing reflex, the digastric or jaw-opening reflex, is also facilitated under the same conditions as the masseteric reflex. If so, it would suggest that NE exerts a somewhat global facilitatory influence, augmenting whatever neuronal system is simultaneously coactivated.

\section{References}

Abercrombie, E. D., and B. L. Jacobs (1987) Single-unit response of noradrenergic neurons in the locus coeruleus of freely-moving cats. I. Acutely presented stressful and nonstressful stimuli. J. Neurosci. 7: 2837-2843.

Aghajanian, G. K., and M. A. Rogawski (1983) The physiological rolc of $\alpha$-adrenoceptors in the CNS: New concepts from single-cell studies. Trends Pharm. Sci. 4: 315-317.

Card, J. P., J. N. Riley, and R. Y. Moore (1986) The motor trigeminal nucleus of the rat: Analysis of neuronal structure and synaptic organization of noradrenergic afferents. J. Comp. Neurol. 250: 469484 . 
Chase, M. H., D. J. McGinty, and M. B. Sterman (1968) Cyclic variations in the amplitude of a brainstem reflex during sleep and wakefulness. Experientia 24: 41-48.

Foote, S. L., F. E. Bloom, and G. Aston-Jones (1983) Nucleus locus coeruleus: New evidence of anatomical and physiological specificity. Physiol. Rev. 63: 844-914.

Glavin, G. B. (1985) Stress and brain noradrenaline: A review. Neurosci. Biobev. Rev. 9: 233-243.

Grzanna, R., W. K. Chee, and E. W. Akeyson (1987) Noradrenergic projections to brain-stem nuclei: Evidence for differential projections from noradrenergic subgroups. J. Comp. Neurol. 263: 76-91.

Jacobs, B. L. (1986) Single-unit activity of locus coeruleus neurons in behaving animals. Prog. Neurobiol. 27: 183-194.

Kidokoro, Y., K. Kubota, S. Shuto, and R. Sumino (1968) Reflex organization of cat masticatory muscle. J. Neurophysiol. 31: 695708.

Leitner, D. S., A. S. Powers, C. L. Stitt, and H. S. Hoffman (1981) Midbrain reticular formation involvement in the inhibition of acoustic startle. Physiol. Behav. 26: 259-268.

Levine, E. S., E. D. Abercrombie, R. B. Bandler, and B. L. Jacobs (1987) Activity of locus coeruleus noradrenergic neurons during defense reaction in behaving cats. Soc. Neurosci. Abstr. 13: 660 .

Rasmussen, K., D. A. Morilak, and B. L. Jacobs (1986) Single-unit activity of locus coeruleus neurons in the freely-moving cat. I. During naturalistic behaviors and in response to simple and complex stimuli. Brain Res. 371: 324-334.
Reiner, P. B. (1985) Clonidine inhibits central noradrenergic neurons in unanesthetized cats. Eur. J. Pharm. 111: 249-257.

Reiner, P. B. (1986) Correlational analysis of central noradrenergic neuronal activity and sympathetic tone in behaving cats. Brain Res. 378: 86-96.

Tanaka, M., Y. Kohno, R. Nakagawa, Y. Ida, S. Takeda, N. Nagasaki, and Y. Noda (1983) Regional characteristics of stress-induced increases in brain noradrenaline release in rats. Pharm. Biochem. Behav. 19: 543-547.

Travers, J. B., and R. Norgren (1983) Afferent projections to the oral motor nuclei in the rat. J. Comp. Neurol. 220: 280-298.

Uchizono, K. (1965) Characteristics of excitatory and inhibitory synapses in the central nervous system of the cat. Nature 207: 642-643.

Vornov, J. J., and J. Sutin (1983) Brainstem projections to the normal and noradrenergically hyperinnervated trigeminal motor nucleus. $\mathrm{J}$. Comp. Neurol. 214: 198-208.

Wamsley, J. K., M. S. Lewis, W. S. Young, and M. J. Kuhar (1981) Autoradiographic localization of muscarinic cholinergic receptors in rat brainstem. J. Neurosci. 1: 176-191.

Wright, C. G., and C. D. Barnes (1972) Audio-spinal responses in decerebrate and choralose anesthetized cats. Brain Res. 36: 307-331.

Wu, M. F., S. S. Suzuki, and J. M. Siegel (1988) Anatomical distribution and response patterns of reticular neurons active in relation to acoustic startle. Brain Res 457: 399-406. 